TRANSACTIONS OF THE

AMERICAN MATHEMATICAL SOCIETY

Volume 364, Number 3, March 2012, Pages 1211-1224

S 0002-9947(2011)05486-3

Article electronically published on October 19, 2011

\title{
EMBEDDED PLATEAU PROBLEM
}

\author{
BARIS COSKUNUZER
}

\begin{abstract}
We show that if $\Gamma$ is a simple closed curve bounding an embedded disk in a closed 3-manifold $M$, then there exists a disk $\Sigma$ in $M$ with boundary $\Gamma$ such that $\Sigma$ minimizes the area among the embedded disks with boundary $\Gamma$. Moreover, $\Sigma$ is smooth, minimal and embedded everywhere except where the boundary $\Gamma$ meets the interior of $\Sigma$. The same result is also valid for homogeneously regular manifolds with sufficiently convex boundary.
\end{abstract}

\section{INTRODUCTION}

The Plateau problem asks the existence of an area minimizing disk for a given simple closed curve in a manifold $M$. This problem was solved for $\mathbf{R}^{3}$ by Douglas [Do], and Rado [Ra in the early 1930s. Later, it was generalized by Morrey for Riemannian manifolds $\mathrm{Mo}$. Then, regularity (nonexistence of branch points) of these solutions was shown by Osserman Os, Gulliver Gu and Alt Al. However, these area minimizing disks may not be embedded, even though the curves bound an embedded disk in the ambient manifold. They might have self-intersections (see Figure 1).

In the following decades, the question of embeddedness of the area minimizing disk was studied: For which curves are the area minimizing disks embedded? The first such condition ensuring the embeddedness of the disk was due to Rado. In the early 1930s, he showed that if the curve can be projected onto a convex curve in a plane, then it bounds a unique embedded minimal disk which is a graph over the plane. Osserman conjectured that if the curve is extreme (lies in the boundary of its convex hull), then the area minimizing disk spanning the curve must be embedded. In the late 1970s, Gulliver and Spruck proved that if the total curvature of an extreme curve is less than $4 \pi$, then the solution to the Plateau problem is embedded [GS]. Later, Almgren-Simon [AS] and Tomi-Tromba [TT] showed the existence of an embedded solution for extreme curves. Then, Meeks and Yau proved the Osserman conjecture in full generality: Any solution to the Plateau problem for an extreme curve must be embedded MY1. Recently, Ekholm, White, and Wienholtz generalized Gulliver-Spruck's embeddedness result by removing extremeness condition from the curves [EWW]. Also, recently, Hass, Lagarias and Thurston [HLT] gave interesting results about the isoperimetric inequalities for embedded disks in $\mathbf{R}^{3}$.

Received by the editors April 28, 2009 and, in revised form, February 25, 2010.

2010 Mathematics Subject Classification. Primary 53A10; Secondary 57M50, 49Q05.

The author was partially supported by EU-FP7 Grant IRG-226062 and TUBITAK Grant $109 \mathrm{~T} 685$.

(C)2011 American Mathematical Society Reverts to public domain 28 years from publication 
On the other hand, a different version of the Plateau problem was studied after the 1960s. This version asks the existence of an area minimizing surface for a given simple closed curve. If there is no restriction on the topological type of the surface, Geometric Measure Theory gives a positive solution for this question. Federer et al. solved the problem and showed the existence of a surface which minimizes area among all surfaces with the given boundary [Fe]. Moreover, any such surface must be embedded for any simple closed curve.

If we come back to the disk case, there is a relevant result about the same question due to Meeks-Yau [MY3. They give a necessary condition for a sufficiently smooth simple closed curve in a 3-manifold to bound an embedded minimal disk. In particular, they showed that for a sufficiently smooth simple closed curve $\Gamma$ in a 3 -manifold $M$, in order to bound a strictly stable embedded minimal disk in $M, \Gamma$ must be an extreme curve in some sense (see Theorem 4.1).

In this paper, we are approaching the embeddedness question from a different direction. Instead of considering the question that "for which curves must the area minimizing disks be embedded?", we analyze the structure of the surface which minimizes area among the embedded disks whose boundary is any given simple closed curve.

Embedded Plateau problem. Let $\Gamma$ be a simple closed curve in a manifold $M$, and let $\Gamma$ bound an embedded disk. Does there exist an embedded minimal disk which minimizes the area among the embedded disks with boundary $\Gamma$ ?

This is the most general case for a curve to bound an embedded minimal disk. This is because if $\Gamma$ does not bound any embedded disk in $M$, then, of course, there is no embedded minimal disk bounding $\Gamma$ at all. Our main result is as follows:

Theorem 3.1. Let $\Gamma$ be a simple closed curve bounding an embedded disk in a closed 3-manifold $M$. Then, there exists a disk $\Sigma$ in $M$ with $\partial \Sigma=\Gamma$ such that $\Sigma$ minimizes the area among all the embedded disks bounding $\Gamma$. Moreover, $\Sigma$ is minimal and smoothly embedded everywhere except where the boundary $\Gamma$ meets the interior of $\Sigma$.

In particular, if $\Sigma$ is as in the theorem, then there is a continuous parametrization $\varphi: D^{2} \rightarrow M$ of $\Sigma$, with $\varphi\left(D^{2}\right)=\Sigma$ and $\varphi\left(\partial D^{2}\right)=\Gamma$, such that $\varphi$ is a smooth embedding on $D^{2}-\varphi^{-1}(\Gamma)$ and the image $\Sigma-\Gamma$ is a minimal surface (see Figure 1). $\lambda=\Gamma \cap \varphi\left(\operatorname{int}\left(D^{2}\right)\right)$ is known as the coincidence set in the literature (see Remark 3.2). If $\lambda=\emptyset$, then $\Sigma$ is a smooth embedded minimal disk in $M$ with boundary $\Gamma$. We call such a disk $\Sigma$ a pseudo-area minimizing disk.

The outline of the technique is summarized as follows: Let $\Gamma$ be any simple closed curve bounding an embedded disk in a manifold $M$. By drilling out a small neighborhood $N_{i}$ of $\Gamma$ and changing the metric in a very small neighborhood of the boundary, we can get a manifold $M_{i}$ with a convex boundary $\partial M_{i}$. For a curve $\Gamma_{i} \subset \partial M_{i}$ homotopic to $\Gamma$ in $M$, there is an area minimizing embedded disk $D_{i}$ in $M_{i}$ with $\partial D_{i}=\Gamma_{i}$ by [MY2]. When $N_{i}$ gets smaller, we get a sequence of embedded disks $\left\{D_{i}\right\}$ in $M$ where the areas of the disks are approaching the minimum area for embedded disks, and $\partial D_{i}=\Gamma_{i} \rightarrow \Gamma$. Then, the idea is to obtain a limiting surface out of this sequence and to analyze its structure.

By using the standard generalizations on $M$ to ensure the embeddedness of the solutions of the Plateau problem in the Meeks-Yau setting, we also give a 


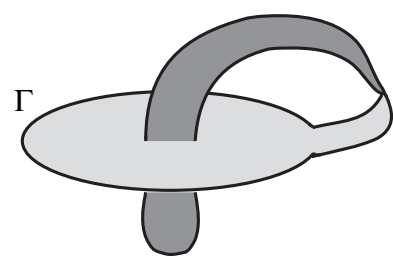

An area minimizing disk

might have transverse self intersections.

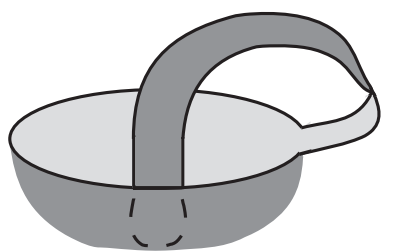

A pseudo-area minimizing disk is embedded everywhere except where $\Gamma$ meet interior.

Figure 1. For a given $\Gamma$, while the area minimizing disks might have transverse self-intersections (left), the pseudo-area minimizing disks have no transverse self-intersection and are embedded everywhere except where the boundary bumps into the interior (right).

generalization of the main result to a homogeneously regular 3-manifold $M$ with sufficiently convex boundary (see Theorem 3.2 ).

On the other hand, by slightly modifying the pseudo-area minimizing disk $\Sigma$ with boundary $\Gamma$, it is easy to get a smooth, embedded disk $\Sigma^{\prime}$ with boundary $\Gamma$ such that $\Sigma^{\prime}$ is minimal everywhere except for a very small region (see Corollary $3.3)$.

The organization of the paper is as follows: In section 2, we cover some basic results which will be used in the remaining part of the paper. In section 3 , we prove the main result. Then in section 4 , we make some final remarks.

\section{Preliminaries}

In this section, we will overview the basic results which we use in the following sections. For more details on these, see [CM] or [HS].

Definition 2.1. Let $M$ be a 3-manifold. A minimal disk in $M$ is a disk whose mean curvature vanishes everywhere. An area minimizing disk in $M$ is a disk which has the smallest area among the disks in $M$ with the same boundary. A pseudo-area minimizing disk in $M$ is a disk which has the smallest area among the embedded disks in $M$ with the same boundary, and has no transverse self-intersection.

Definition 2.2 ([HS $)$. Let $M$ be a compact Riemannian 3-manifold with boundary. Then $M$ is mean convex (or $M$ has sufficiently convex boundary) if the following conditions hold:

- $\partial M$ is piecewise smooth.

- Each smooth subsurface of $\partial M$ has nonnegative curvature with respect to the inward normal.

- There exists a Riemannian manifold $N$ such that $M$ is isometric to a submanifold of $N$ and each smooth subsurface $S$ of $\partial M$ extends to a smooth embedded surface $S^{\prime}$ in $N$ such that $S^{\prime} \cap M=S$.

Definition 2.3 ( $\mathrm{MY1}$, [HS $)$. Let $M$ be a Riemannian 3-manifold. Then $M$ is homogeneously regular if the sectional curvature is bounded above and the injectivity radius is bounded away from 0 . 
In this paper, we will use the following definition for extreme curves. Note that this definition is different from the one in the literature (lying in the boundary of its convex hull), and our definition is more general than the other one.

Definition 2.4. $\Gamma \subset M$ is an extreme curve if it is a curve in the boundary of a mean convex submanifold in $M$.

Now, we state the main facts which we use in the following sections.

Lemma 2.1 ([MY1, MY2]). Let $M$ be a compact manifold with sufficiently convex boundary, and let $\Gamma$ be a nullhomotopic simple closed curve in $\partial M$. Then, there exists an area minimizing disk $D$ in $M$ with $\partial D=\Gamma$. Moreover, unless $D \subset \partial M$, all such disks are properly embedded (i.e. the boundary of the disk is in the boundary of the manifold) in $M$ and they are pairwise disjoint.

Now, we state two lemmas due to Hass and Scott [HS], which we use in the following sections.

Lemma 2.2 ([ HS Lemma 3.1]). Let $M$ be a closed Riemannian 3-manifold. Then, there exists an $\epsilon>0$ such that for any $x \in M$, the ball $B_{\epsilon}(x)$ of radius $\epsilon$ about $x$ in $M$ has the following property: If $\Gamma \subset \partial B_{\epsilon}(x)$ is a simple closed curve, and if $D$ is an area minimizing disk in $M$ with $\partial D=\Gamma$, then $D$ is properly embedded in $B_{\epsilon}(x)$.

Lemma 2.3 ([HS, Lemma 3.6]). Let $M$ be a compact Riemannian 3-manifold with strictly convex boundary. Let $\left\{D_{i}\right\}$ be a sequence of properly embedded area minimizing disks in $M$ which have uniformly bounded area. Then there is a subsequence $\left\{D_{i_{j}}\right\}$ which converges to a collection of properly embedded area minimizing disks. If $\left\{D_{i}\right\}$ has a limit point, then the collection is not empty.

\section{MAIN RESUlT}

In this section, we will prove the main result of the paper.

Let $\Gamma$ be a simple closed curve which is the boundary of an embedded disk $E$ in a closed Riemannian 3-manifold $M$.

First, we will construct a sequence of embedded almost area minimizing disks $\left\{D_{i}\right\}$ in $M$ with $\partial D_{i}=\Gamma_{i}$ and $\Gamma_{i} \rightarrow \Gamma$ by using the techniques of Calegari and Gabai in [Ga] and [CG]. Then, by taking the limit of this sequence as in [Ga] and $[\mathrm{HS}$, we will get a limit object $\Delta$. Then, by analyzing this object, we will show the main result of the paper.

3.1. The sequence. Take a sequence of open solid tori $\left\{N_{i}\right\}$ which are neighborhoods of $\Gamma$. That is, fix a sufficiently small $\epsilon>0$, and let $N_{i}=N_{\frac{\epsilon}{i}}(\Gamma)$, where $N_{\epsilon}($. represents the open $\epsilon$-neighborhood in $M$. Then $N_{1} \supset N_{2} \supset \cdots \supset N_{i} \supset N_{i+1} \supset \ldots$ and $\bigcap_{i=1}^{\infty} N_{i}=\Gamma$.

Now, let $M_{i}=M-N_{i}$. Clearly, $\left\{M_{i}\right\}$ are compact 3-manifolds with torus boundary. Moreover, $M_{1} \subset M_{2} \subset \ldots \subset M_{i} \subset M_{i+1} \subset \ldots$ and $\bigcup_{i=1}^{\infty} M_{i}=M-\Gamma$. Also, note that for $\delta<\epsilon, \partial \overline{N_{\delta}(\Gamma)}$ is a torus, and let $F:(0, \epsilon] \rightarrow \mathbf{R}$ be a function such that $F(\delta)=\left|\partial \overline{N_{\delta}(\Gamma)}\right|$, where $|$.$| represents the area in M$. Since $\partial \overline{N_{\delta}(\Gamma)}$ degenerates into $\Gamma$ as $\delta \rightarrow 0$, then $F(\delta) \rightarrow 0$ as $\delta \rightarrow 0$. Hence, $\left|\partial M_{i}\right| \rightarrow 0$ as $i \rightarrow \infty$.

Now, we will construct a sequence of area minimizing disks in $M$. Let $E$ be the disk in $M$ bounding $\Gamma$. Modify $E$ if necessary so that $E$ is transverse to $\partial M_{i}$. Then, let $\widehat{\Gamma}_{i}=E \cap \partial M_{i}$. In other words, for each $i$, let $\widehat{\Gamma}_{i}$ be a simple closed curve in $\partial M_{i}=\partial N_{i}$ which is isotopic to the core curve $\Gamma$ in the solid torus $N_{i}$. 
Consider the manifolds with torus boundary $M_{i} \subset M_{i+1}$. Change the metric of $M_{i+1}$ in $M_{i+1}-M_{i}$ so that $M_{i+1}$ becomes a compact manifold with sufficiently convex boundary, say $\widehat{M}_{i+1}\left(M_{i+1}\right.$ with a new metric). Note that the new metric is the same as the original metric of $M$ in the $M_{i}$ part. As $\widehat{M}_{i+1}$ is mean convex, by Lemma 2.1 , there exists an area minimizing disk $\widehat{D}_{i+1}$ in $\widehat{M}_{i+1}$ with $\partial \widehat{D}_{i+1}=\widehat{\Gamma}_{i+1}$ (see Figure 2).

Now, consider the intersection $\partial M_{i} \cap \widehat{D}_{i+1}$. By modifying $M_{i}$ if necessary, we can assume the intersection is transverse, and it is a collection of simple closed curves. By construction, the curves in the intersection are either essential in $\partial M_{i}$ and isotopic to $\Gamma$ in $M$, or not essential in $\Gamma$. Let $\left\{\alpha_{1}, \alpha_{2}, \ldots, \alpha_{n}\right\}$ be essential curves, and let $\left\{\beta_{1}, \beta_{2}, \ldots, \beta_{m}\right\}$ be the nonessential ones. By construction, we know that $n \geq 1$ and $m \geq 0$. Without loss of generality, let $\alpha_{1}$ be the innermost curve in $\widehat{D}_{i+1}$ among $\left\{\alpha_{1}, \alpha_{2}, \ldots, \alpha_{n}\right\}$. Let $E_{i}$ be the subdisk in $\widehat{D}_{i+1}$ with $\partial E_{i}=\alpha_{1}$.

Now, consider that $\partial E_{i} \subset \partial M_{i}$ and $E_{i} \subset M_{i+1}$. If $E_{i} \subset M_{i}$, then define the $i^{t h}$ element of the desired sequence $D_{i}=E_{i}$. Otherwise, $E_{i} \cap\left(M_{i+1}-M_{i}\right) \neq \emptyset$ and consists of planar surfaces whose boundary is some curves in $\left\{\beta_{1}, \beta_{2}, \ldots, \beta_{m}\right\}$. Now replace these planar surfaces in $E_{i}$ with the isotopic surface in $\partial M_{i}$ with the same boundary. Then, smooth out the corners into $M_{i}$ and push the parts in $\partial M_{i}$ into $M_{i}$ so that surgered $E_{i}$, say $\widehat{E}_{i}$, becomes a disk in $M_{i}$. Hence, define $\Gamma_{i}$ as $\alpha_{1}$, and $D_{i}$ as $\widehat{E}_{i}$.

By construction $\left\{D_{i}\right\}$ has the following properties:

- $D_{i}$ is a properly embedded smooth disk in $M_{i}$, i.e. $\partial D_{i}=\Gamma_{i}=D_{i} \cap \partial M_{i}$.

- $\left|D_{i}\right|<T_{i}+2\left|\partial M_{i}\right|$, where $T_{i}$ is the infimum of the areas of the embedded disks in $M$ with boundary $\Gamma_{i}$.

By construction, the first property is clear. To see the second property, first consider that $E_{i}$ is the area minimizing disk in $\widehat{M}_{i+1}$, and the metric of $\widehat{M}_{i+1}$ coincides with the original metric on $M$ in $M_{i}$. Clearly, $T_{i}^{\prime}<T_{i}+\left|\partial M_{i}\right|$, where $T_{i}^{\prime}$ is the infimum of the areas of the embedded disks in $M_{i}$ with boundary $\Gamma_{i}$, as $D_{i}$ is obtained by modifying $E_{i} \cap M_{i}$, by patching the missing parts from $\partial M_{i}$ and smoothing out corners. By [MY2], smoothing out the folding curves decreases the area; hence $\left|D_{i}\right| \leq\left|E_{i} \cap M_{i}\right|+\left|\partial M_{i}\right|$. Since $\left|E_{i} \cap M_{i}\right| \leq T_{i}^{\prime}$, the second property follows.

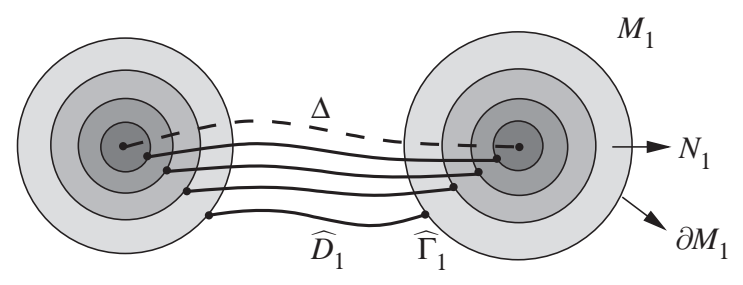

Figure 2. For any $i, M_{i}=M-N_{i}$; then $N_{i} \supset N_{i+1}$, and $M_{i} \subset$ $M_{i+1} \cdot \widehat{\Gamma}_{i} \subset \partial M_{i}$ is a simple closed curve isotopic to $\Gamma$ in $M\left(\left\{\widehat{\Gamma}_{i}\right\}\right.$ are shown as pairs of points, and $\Gamma$ is shown as the innermost pair of points in the figure). $\widehat{D}_{i}$ is an area minimizing disk in $\widehat{M}_{i}\left(M_{i}\right.$ with modified metric) with $\partial \widehat{D}_{i}=\widehat{\Gamma}_{i}$. 
It is clear that $D_{i}$ may not be area minimizing in $M$; however, with the second property, they can be thought of as almost area minimizing in $M$. Note that $E_{i} \cap M_{i} \subset D_{i}$ is area minimizing in $M$, so only the patched parts of $D_{i}$ (replacements of $\left.E_{i} \cap\left(M_{i+1}-M_{i}\right)\right)$ are not area minimizing, which are a very small part of $D_{i}$. This is because it is reasonable to think of $D_{i}$ as almost area minimizing.

Now, let $T$ be the infimum of the areas of the embedded disks in $M$ with boundary $\Gamma$. Let $A_{i}$ be the infimum of the areas of embedded annuli with boundary $\Gamma \cup \Gamma_{i}$. By construction, $T_{i} \leq T+A_{i}$ and $T \leq T_{i}+A_{i}$. Since $\Gamma_{i} \rightarrow \Gamma, A_{i} \rightarrow 0$ as $i \rightarrow \infty$. This implies $T_{i} \rightarrow T$ as $i \rightarrow \infty$. Hence, by the second property and $\left|\partial M_{i}\right| \rightarrow 0$, $\left|D_{i}\right| \rightarrow T$ as $i \rightarrow \infty$.

To sum up, we constructed a sequence of embedded smooth disks $\left\{D_{i}\right\}$ in $M$ such that $\partial D_{i}=\Gamma_{i} \subset \partial M_{i}$ with $\Gamma_{i} \rightarrow \Gamma$ and $\left|D_{i}\right| \rightarrow T$ as $i \rightarrow \infty$, where $T$ is the infimum of the areas of the embedded disks in $M$ with boundary $\Gamma$.

3.2. The limit. In this section, by using the techniques of $\mathrm{Ga}$ and $\mathrm{HS}$, from the sequence $\left\{D_{i}\right\}$ of almost area minimizing embedded disks in $M$, we will get a limit object $\Delta$ which can be thought of as a special topological limit. Then, by using this object, we will prove the main result of the paper. The following is a modified version of a definition of a special topological limit due to Gabai Ga].

Definition 3.1. A collection of pairwise disjoint embedded surfaces $\Delta$ in a Riemannian manifold $M$ is called the topological limit of the sequence $\left\{D_{i}\right\}$ of embedded surfaces in $M$ if

- $\Delta=\left\{x=\lim x_{i} \mid x_{i} \in D_{i},\left\{x_{i}\right\}\right.$ is a convergent sequence in $\left.M\right\}$

- $\Delta=\left\{x=\lim x_{n_{i}} \mid x_{i} \in D_{i},\left\{x_{i}\right\}\right.$ has a convergent subsequence $\left\{x_{n_{i}}\right\}$ in $\left.M\right\}$

In other words, the sequence $\left\{D_{i}\right\}$ is such that the set of the limits of all $\left\{x_{i}\right\}$ with $x_{i} \in M$ and the set of the limits of the subsequences are the same. This is an essential condition on $\Delta$ to be a collection of pairwise disjoint embedded surfaces. Otherwise, one might simply take a sequence such that $D_{2 i+1}=\Sigma_{1}$ and $D_{2 i}=\Sigma_{2}$, where $\Sigma_{1}$ and $\Sigma_{2}$ are intersecting disks. Then, without the first condition ( $\Delta$ being just the union of limit points), $\Delta=\Sigma_{1} \cup \Sigma_{2}$ in this case, which is not a collection of pairwise disjoint embedded disks. However, the first condition forces $\Delta$ to be either $\Sigma_{1}$ or $\Sigma_{2}$, not the union of them. By similar reasons, this condition is also important to ensure the embeddedness of the disks in the collection $\Delta$.

Now, we will show that there is a subsequence of the sequence constructed in the previous part which gives a topological limit $\Delta$. Then, by showing that the limit $\Delta$ is a collection of disks, we will prove the main result of the paper.

Theorem 3.1. Let $\Gamma$ be a simple closed curve bounding an embedded disk in a closed 3-manifold $M$. Then, there exists a disk $\Sigma$ in $M$ with $\partial \Sigma=\Gamma$ such that $\Sigma$ minimizes the area among the embedded disks bounding $\Gamma$. Moreover, $\Sigma$ is minimal and smoothly embedded everywhere except where the boundary $\Gamma$ meets the interior of $\Sigma$.

Proof. Let $\left\{D_{i}\right\}$ be as defined in the previous part. We abuse the notation by taking $\left\{D_{i}\right\}$ as a sequence of open disks. Our aim is to get a convergence as in Definition 3.1 for an appropriate subsequence of $\left\{D_{i}\right\}$. 
Step 0. After passing to a subsequence of $\left\{D_{i}\right\}$, the following holds:

$$
\begin{aligned}
\Delta & =\left\{x=\lim x_{i} \mid x_{i} \in D_{i},\left\{x_{i}\right\} \text { is a convergent sequence in } M\right\} \\
& =\left\{x=\lim x_{n_{i}} \mid x_{i} \in D_{i},\left\{x_{i}\right\} \text { has a convergent subsequence }\left\{x_{n_{i}}\right\} \text { in } M\right\} .
\end{aligned}
$$

Proof. For each $j$ subdivide $M$ into a finite number of closed regions such that the $j+1^{s t}$ subdivision is a subdivision of the $j^{t h}$ one. Also, let the mesh of these subdivisions converge to 0 . In other words, let $B_{k}^{j}$ be the $k^{\text {th }}$ region of the $j^{\text {th }}$ subdivision and $M=\bigcup_{k=1}^{n_{j}} B_{k}^{j}$, where $B_{i}^{j-1}=B_{i_{1}}^{j} \cup \ldots \cup B_{i_{r}}^{j}$. Also, for any $j, k$, $\operatorname{diam}\left(B_{k}^{j}\right)<C_{j}$, where $C_{j} \rightarrow 0$ as $j \rightarrow \infty$.

Now, choose a subsequence of $\left\{D_{i}\right\}$ such that if $i \geq j$ and $D_{i} \cap B_{k}^{j} \neq \emptyset$, then for any $k>i, D_{k} \cap B_{r}^{j} \neq \emptyset$. By abuse of notation, replace this subsequence with the original sequence $\left\{D_{i}\right\}$. Now, for this new sequence, for any $x=\lim x_{n_{i}}$ where $x_{n_{i}} \in D_{n_{i}}$, by construction, there is a convergent sequence $\left\{x_{i}\right\}$ with $x_{i} \in D_{i}$ such that $x=\lim x_{i}$. Hence, Step 0 follows.

Step 1. $\Delta$ is not empty.

Proof. In order to show that $\Delta$ is nonempty, it will suffice to construct a convergent sequence $\left\{x_{n_{i}}\right\}$ in $M$ with $x_{n_{i}} \in D_{n_{i}}$. Now, consider a meridian curve $\gamma^{\prime}$ of $\partial M_{1}$ (think of $\Gamma_{1}$ as the longitude of $\partial M_{1}$ ). Push $\gamma^{\prime}$ into $M_{1}$ a little bit (a small isotopy), and denote the new curve by $\gamma$ in $M_{1}$. Now, $\gamma$ links $\Gamma$ and $\Gamma_{i}$ in $M$ for all $i$. Hence, for any $i, D_{i} \cap \gamma$ is not empty. Let $x_{i} \in D_{i} \cap \gamma$. Since $\gamma$ is a simple closed curve, the sequence $\left\{x_{i}\right\}$ must have a convergent subsequence. Hence, $\Delta$ is nonempty.

Step 2. Let $Z=\left\{z_{i}\right\}$ be a countable dense subset of $\Delta$, where $Z \cap \Gamma=\emptyset$. Then, after passing to a subsequence of $\left\{D_{j}\right\}$, the following holds. For any $i$, there exists a sequence $\left\{E_{j}^{i}\right\}$ of embedded disks $E_{j}^{i} \subset D_{j}$ which converges to a smoothly embedded disk $E_{i}$ such that $z_{i} \in E_{i}$.

Proof. As $M$ is a closed manifold, by Lemma 2.2, there exists a $\rho>0$ such that for any $x \in M$, if $\Gamma \subset \partial B_{\rho}(x)$, then any area minimizing disk $D$ in $M$ with boundary $\Gamma$ must be in $B_{\rho}(x)$.

Now, let $Z_{1}=\left\{z_{i} \in Z \mid d\left(z_{i}, \Gamma\right)<2 \rho\right\}$ and $Z_{2}=Z-Z_{1}$. Also, let $\rho_{i}=\frac{d\left(z_{i}, \Gamma\right)}{2}$ for any $z_{i} \in Z_{1}$. Now, we claim that for any $z_{i} \in Z_{1}$, there is an embedded disk $E_{i}$ in $\Delta$ with $z_{i} \subset E_{i}$ and $\partial \bar{E}_{i} \cap B_{\rho_{i}}\left(z_{i}\right)=\emptyset$. We also claim that for $z_{i} \in Z_{2}$, there is an embedded disk $E_{i}$ in $\Delta$ with $z_{i} \subset E_{i}$ and $\partial \bar{E}_{i} \cap B_{\rho}\left(z_{i}\right)=\emptyset$.

Now, fix $z_{i} \in Z_{2}$. Since $z_{i} \in \Delta$, there exists a sequence $\left\{x_{j}^{i}\right\}$ with $x_{j}^{i} \in D_{j}$ and $x_{j}^{i} \rightarrow z_{i}$. By deleting the tangential points in the intersection, and by modifying $\rho$ if necessary, we can assume that $\partial B_{\rho}\left(z_{i}\right)$ is transverse to $\left\{D_{j}\right\}$. Let $E_{j}^{i}=B_{\rho}\left(z_{i}\right) \cap D_{j}$. For sufficiently large $j$, being away from $\partial M_{j}$, the almost area minimizing disk $D_{j}$ is area minimizing near $z_{i}$ and hence $E_{j}^{i}$ is an area minimizing disk in $B_{\rho}\left(z_{i}\right)$ by Lemma 2.2. Hence $\left|E_{j}^{i}\right|<\frac{1}{2}\left|\partial B_{\rho}\left(z_{i}\right)\right|$ for any $j$, and the sequence of properly embedded area minimizing disks in $B_{\rho}\left(z_{i}\right)$ have uniformly bounded area. Also, since $M$ is closed, we can assume that $B_{\rho}\left(z_{i}\right)$ has a strictly convex boundary, by taking a smaller $\rho$ if necessary (since $M$ is compact, there exists $R$ such that for any $0<r<R, B_{r}(x)$ has a strictly convex boundary for any $\left.x \in M\right)$. Hence, by Lemma $2.3,\left\{E_{j}^{i}\right\}$ has a subsequence converging to $E_{i}$, where $E_{i}$ is the area minimizing disk in $M$ with $z_{i} \in E_{i}$. By using the diagonal subsequence argument, we can modify our sequence $\left\{D_{i}\right\}$ accordingly. In other words, for each $z_{i}$, use the 
above argument and get a diagonal subsequence, and call this new sequence $\left\{D_{i}\right\}$ again (abuse of notation), and define $\Delta$ for this new sequence.

Similarly, fix $z_{i} \in Z_{1}$. As above, let $\left\{x_{j}^{i}\right\}$ be a sequence with $x_{j}^{i} \in D_{j}$ and $x_{j}^{i} \rightarrow z_{i}$, and $E_{j}^{i}=B_{\rho_{i}}\left(z_{i}\right) \cap D_{j}$. By construction, $\rho_{i} \leq \rho$ for any $i$. Again for sufficiently large $j$, being away from $\partial M_{j}$, an almost area minimizing disk $D_{j}$ is area minimizing near $z_{i}$ and hence $E_{j}^{i}$ is an area minimizing disk in $B_{\rho_{i}}\left(z_{i}\right)$ by Lemma 2.2. As before, by Lemma $2.3,\left\{E_{j}^{i}\right\}$ has a subsequence converging to $E_{i}$, where $E_{i}$ is the area minimizing disk in $M$ with $z_{i} \in E_{i}$. Continuing with the diagonal subsequence argument for each $z_{i}$, we get a subsequence of $\left\{D_{i}\right\}$ with the required properties.

Hence, with the above construction, we get $\bigcup_{z_{i} \in Z} E_{i}=\Delta-\Gamma$. Now, we will analyze the structure of the limit object $\Delta$. In particular, we will prove that $\Delta$ is a union of "disks" which minimize area among the embedded disks. Now, clearly $\Gamma \subset \Delta$. We want to specify some parts of $\Gamma$ which bump into the interior of $\Delta$. In other words, define the coincidence set $\lambda \subset \Gamma$ such that

$\lambda=\left\{x \in \Gamma \mid \exists \rho_{x}>0, \exists\left\{y_{j}\right\}\right.$ with $y_{j} \in D_{j}$ and $\hat{d}_{j}\left(y_{j}, \Gamma_{j}\right)>\rho_{x}$ such that $\left.y_{j} \rightarrow x\right\}$.

Here, $\hat{d}_{j}$ is the induced path metric on $D_{j}$. In other words, a point in the boundary $\Gamma$ is in the coincidence set $\lambda$ if there exists a sequence $\left\{z_{j}\right\}$ in $\Delta$ which is away from the boundary $\Gamma$ and $z_{j} \rightarrow x$. Hence by definition, the coincidence set $\lambda$ corresponds to some closed subsegments of $\Gamma$ which meet the interior part of $\Delta$. It can be thought of as the defective parts of the embedded minimal disks we are constructing. Note that $\lambda$ might be empty.

Step 3. $\Delta-\Gamma$ is a minimal surface in $M$.

Proof. By Step 2, for each $z_{i} \in Z$, there is an embedded disk $E_{i} \subset \Delta$. Now, if $x \in E_{i} \cap E_{j}$, then they must coincide in a neighborhood of $x$. Otherwise, since $E_{i}$ and $E_{j}$ are minimal disks, they must cross transversely near $x$ [HS. However, by construction, this would imply that $D_{i}$ is not embedded for sufficiently large $i$. Hence, the $E_{i}$ 's are either pairwise disjoint or locally coincide. As $\left\{z_{i}\right\}$ is a dense subset of $\Delta$, by Step 2, for any $x \in \Delta-\Gamma$, we can find a neighborhood of $x$ in $\Delta$, say $E_{x}$, which is an open minimal disk. Minimality comes from being locally area minimizing. Hence, $\Delta-\Gamma=\bigcup_{x \in \Delta-\Gamma} E_{x}$. This shows that each component $\Sigma_{i}^{\prime}$ of $\Delta-\Gamma$ is a surface.

Step $4 . \Delta$ is a collection of disks which minimize area among the embedded disks.

Proof. Now, we will show that for each component $\Sigma_{i}^{\prime}$ of $\Delta-\Gamma, \Sigma_{i}=\Sigma_{i}^{\prime} \cup \Gamma$ is a pseudo-area minimizing disk in $M$ where $\Sigma_{i}-\Gamma$ is a smooth minimal surface. In other words, we will show that there is a continuous map $\varphi_{i}: D^{2} \rightarrow M$ such that $\varphi\left(\partial D^{2}\right)=\Gamma$ and $\varphi\left(D^{2}\right)=\Sigma_{i}$, where $\varphi$ is an embedding except at $\varphi^{-1}(\lambda)$. That is, $\Sigma_{i} \subset M$ is the image of a continuous map from a disk where it fails to be an embedding only at the coincidence set $\lambda$.

Let $\alpha$ be a simple closed curve in $\Sigma_{i}^{\prime}$. Let $A$ be a neighborhood of $\alpha$ which is a very thin annulus in $\Sigma_{i}^{\prime}$. Now, let $g: D \rightarrow A$ be an isometric immersion of a disk $D$ into $\Sigma_{i}$, where $D$ is a very long thin rectangle with $g(D)=A$. Also, assume that $|D|>C$, where $C$ is a constant with $C>\left|D_{i}\right|$ for any $i$. Existence of such a $C$ comes from the construction as $D_{i}$ is an area minimizing disk in $M_{i}$, and 
$\left|D_{i}\right|<\left|A_{i}\right|+\left|D_{1}\right|$ where $A_{i}$ is a very thin annulus with $\partial A_{i}=\Gamma_{1} \cup \Gamma_{i}$. Since we can find a uniform bound for $\left|A_{i}\right|$, the existence of $C$ follows.

Now, as $D_{i} \rightarrow \Delta$, and $A \subset \Delta-\Gamma$, we can find isometric immersions $g_{i}: D^{2} \rightarrow D_{i}$ such that $g_{i} \rightarrow g$ in the $C^{\infty}$ topology. Now, there are two cases. Either $g_{i}\left(D^{2}\right) \subset$ $D_{i}$ is also a thin annulus $A_{i}$ in $D_{i}$ approximating $A$, or $g_{i}\left(D^{2}\right)$ is an embedded disk in $D_{i}$ which is spiraling around $A$. In the latter case, this would mean that $\left|D_{i}\right|>|D|>C$, which is a contradiction. Hence, $g_{i}\left(D^{2}\right)=A_{i}$. Then, we can choose a suitable essential simple closed curve $\beta_{i}$ in each annulus $A_{i}\left(\beta_{i}\right.$ is a core curve of the annulus $A_{i}$ ) such that the sequence $\left\{\beta_{i}\right\}$ converges to $\alpha$, i.e. $\beta_{i} \rightarrow \alpha$. Let $F_{i}$ be the disks in $D_{i}$ with $\partial F_{i}=\beta_{i}$.

Now, further assume that $\alpha$ separates $\Sigma_{i}$ into two parts, say $S_{1}$ and let $S_{2}$, and let $\Gamma \subset S_{2}$. In other words, $S_{1} \subset \Sigma_{i}$ and $\partial S_{1}=\alpha$ with $S_{1} \cap \lambda=\emptyset$. Being away from $\Gamma$, hence from the coincidence set $\lambda$, this implies that the disks $\left\{F_{i}\right\}$ are area minimizing disks (not almost area minimizing). Hence, as in Lemma 3.3 of Ga], the sequence of disks $\left\{F_{i}\right\}$ converges to a disk $\Omega$ in $\Sigma_{i}$ with $\partial \Omega=\alpha$; i.e. $S_{1}=\Omega$ is a smooth minimal disk in $M$. Hence, we show that for any separating simple closed curve $\alpha$ in $\Sigma_{i}$ with $S_{1} \cap \lambda=\emptyset$, there is a smooth disk $\Omega$ in $\Sigma_{i}^{\prime}$ with $\partial \Omega=\alpha$.

Now, by choosing a suitable sequence of simple closed curves $\left\{\alpha_{n}\right\}$ in $\Sigma_{i}$, we can exhaust $\Sigma_{i}$ with disks $\Omega_{n}$ such that $\Omega_{1} \subset \Omega_{2} \subset \ldots \subset \Omega_{n} \subset \ldots$ with $\Sigma_{i}=\bigcup_{n} \Omega_{n}$, where $\Omega_{n}$ is a disk in $\Sigma_{i}$ with $\partial \Omega_{n}=\alpha_{n}$ and $\Omega_{n} \cap \lambda=\emptyset$. This means that $\alpha_{n} \rightarrow \Gamma \cup l \cup \lambda$, where $l$ is a collection of line segments $\left\{l_{j}^{k}\right\}$ in $\Sigma_{i}$ which connects $\Gamma$ with one of the endpoints of the components $\left\{\lambda_{k}\right\}$ of $\lambda$. In particular, for each component $\lambda_{k}$ of $\lambda$, let $n_{k}$ be the number of line segments $\left\{l_{1}^{k}, l_{2}^{k}, \ldots, l_{n_{k}}^{k}\right\}$ connecting the component $\lambda_{k}$ to the $\Gamma$. Then $n_{k}$ is the number of the local sheets of $\Sigma_{i}$ near $\lambda_{k}$, i.e., the components of $N\left(\lambda_{k}\right) \cap \Omega_{n}$, where $N\left(\lambda_{k}\right)$ is a sufficiently small neighborhood of $\lambda_{k}$ in $M$ and $n$ is sufficiently large.

Since the sequence of the disks $\left\{\Omega_{n}\right\}$ exhausting $\Sigma_{i}$ does not contain $\lambda, \lambda$ is in the component $\Sigma_{i}-\Omega_{n}$ which also contains $\Gamma$. Hence, for sufficiently large $n$, $\Sigma_{i}-\Omega_{n}$ is the union of some thin neighborhood of $\Gamma$ and some thin strips around line segments $\left\{l_{j}^{k}\right\}$ connecting $\Gamma$ to $\lambda$, as $\Omega_{n}$ is exhausting $\Sigma_{i}$. Hence, $\alpha_{n}$ curves approach $l \cup \lambda$ from both sides and $\partial \Omega_{n}=\alpha_{n} \rightarrow \Gamma \cup l \cup \lambda$. Hence, we can get a continuous parametrization $\hat{\varphi}: D^{2} \rightarrow M$ with $\hat{\varphi}\left(D^{2}\right)=\Sigma_{i}$ and $\hat{\varphi}\left(\partial D^{2}\right)=\Gamma \cup l \cup \lambda$.

Now, our aim is to get a continuous map $\varphi: D^{2} \rightarrow M$ with $\varphi\left(D^{2}\right)=\Sigma_{i}$ and $\varphi\left(\partial D^{2}\right)=\Gamma$ by modifying $\hat{\varphi}$. Fix $k_{0}$ and $j_{0}$. Consider $l_{j_{0}}^{k_{0}} \cup \lambda_{k_{0}}$. Let $U=N\left(l_{j_{0}}^{k_{0}} \cup \lambda_{k_{0}}\right)$ be a very small neighborhood of $l_{j_{0}}^{k_{0}} \cup \lambda_{k_{0}}$ in $\Sigma_{i}$ such that $U \cap l_{j}^{k}=\emptyset$ for any $(k, j) \neq\left(k_{0}, j_{0}\right)$. Consider $\hat{\varphi}^{-1}(U) \subset D^{2}$. Let $V$ be the component of $\hat{\varphi}^{-1}(U)$ in $D^{2}$ which contains the segment $\hat{\varphi}^{-1}\left(l_{j_{0}}^{k_{0}}\right) \subset \partial D^{2}$. Hence, $\left.\hat{\varphi}\right|_{V}: V \rightarrow U$ and $\hat{\varphi}\left(V \cap \partial D^{2}\right) \subset \Gamma \cup l_{j_{0}}^{k_{0}} \cup \lambda_{k}$.

Now, we can continuously modify $\hat{\varphi}$ in $V$ into a continuous map $\varphi$ so that $\left.\varphi\right|_{V}: V \rightarrow U$ is a continuous embedding with $\varphi\left(\partial \bar{V} \cap \operatorname{int}\left(D^{2}\right)\right)=\hat{\varphi}\left(\partial \bar{V} \cap \operatorname{int}\left(D^{2}\right)\right)$ and $\varphi\left(V \cap \partial D^{2}\right) \subset \Gamma$. To see this, one can define a continuous family of maps $\hat{\varphi}_{t}: V \rightarrow U$ with $0 \leq t \leq 1$ such that $\hat{\varphi}_{0}=\left.\hat{\varphi}\right|_{V}$ and $\hat{\varphi}_{t}\left(V \cap \partial D^{2}\right) \subset \Gamma \cup\left(l_{j_{0}}^{k_{0}} \cup \lambda_{k_{0}}\right)_{t}$. Here, $\left(l_{j_{0}}^{k_{0}} \cup \lambda_{k_{0}}\right)_{t}$ is a subsegment in $l_{j_{0}}^{k_{0}} \cup \lambda_{k_{0}}$ which is getting smaller as $t$ increases, and finally $\left(l_{j_{0}}^{k_{0}} \cup \lambda_{k_{0}}\right)_{1}$ is the endpoint of $l_{j_{0}}^{k_{0}} \cup \lambda_{k_{0}}$ in $\Gamma$. Intuitively, this continuous deformation of the parametrizations corresponds to pushing $l_{j_{0}}^{k_{0}} \cup \lambda_{k}$ into $\Gamma$ in $\Sigma_{i}$. Since, $U=N\left(l_{j_{0}}^{k_{0}} \cup \lambda_{k_{0}}\right)$ is disjoint from other line segments $l_{j}^{k}$, one can modify $\hat{\varphi}$ 
for each $l_{j}^{k}$. Finally, we get a continuous map $\varphi: D^{2} \rightarrow M$ with $\varphi\left(D^{2}\right)=\Sigma_{i}$ and $\varphi\left(\partial D^{2}\right)=\Gamma$.

Even though we obtained $\varphi$ from $\hat{\varphi}$, which may not be smooth along $l \cup \lambda$, since the choice of $l$ is arbitrary, and $\Sigma_{i}-\Gamma$ is smooth by previous steps, $\varphi$ can be chosen as a smooth embedding on $D^{2}-\varphi^{-1}(\Gamma)$ by construction. If the coincidence set $\lambda=\Gamma \cap \varphi\left(\operatorname{int}\left(D^{2}\right)\right)$ is empty, then $\Sigma_{i}$ is an embedded minimal disk in $M$ with $\partial \Sigma_{i}=\Gamma$. Otherwise, the disk $\Sigma_{i}$ might fail to be smooth on $\lambda$. Note also that the restriction of $\varphi$ to the interior of $D^{2}$ may not be an embedding either, if $n_{k}>1$ for some $k$ (see Section 4).

Now, we claim that $\Sigma_{i}$ is minimizing area among the embedded disks with boundary $\Gamma$. Otherwise, there is a compact subdisk $E$ of $\Sigma_{i}$ which is not area minimizing among the embedded disks. Then there is an embedded disk $E^{\prime}$ in $M$ with smaller area. Let $|E|-\left|E^{\prime}\right|=\xi$. By construction, since $D_{i} \rightarrow \Delta$, we can find sufficiently close disks in the sequence $\left\{D_{i}\right\}$ to $\Sigma_{i}$ such that there is a subdisk $E^{\prime \prime} \subset D_{i}$ with ||$E|-| E^{\prime \prime}||<\frac{\xi}{2}$ and $|\mathcal{A}|<\frac{\xi}{2}$, where $\mathcal{A}$ is an annulus with boundary $\partial E \cup \partial E^{\prime \prime}$. However, this implies that $\left|E^{\prime}\right|+|\mathcal{A}|<\left|E^{\prime \prime}\right|$. This is a contradiction as $E^{\prime} \subset D_{i}$ is area minimizing among the embedded disks with the same boundary. So, $\Sigma_{i}$ is also area minimizing among the embedded disks with the same boundary. Hence, Step 4 follows.

Hence, by taking one of the $\Sigma_{i}$ pieces in $\Delta$ given by Step 4, we get a continuous map $\varphi: D^{2} \rightarrow M$ with $\varphi\left(D^{2}\right)=\Sigma_{i}$ and $\varphi\left(\partial D^{2}\right)=\Gamma$. Moreover, $\varphi$ is a smooth embedding on $D^{2}-\varphi^{-1}(\Gamma)$, and $\Sigma_{i}-\Gamma$ is an embedded minimal surface. The proof follows.

Remark 3.1. This theorem shows that for a given simple closed curve $\Gamma$ bounding a disk in a closed 3-manifold, there exists a pseudo-area minimizing disk $\Sigma$ in $M$ with $\partial \Sigma=\Gamma$. Moreover, the theorem gives the structure of the pseudo-area minimizing disks: A pseudo-area minimizing disk $\Sigma$ may not be an embedded disk, but it can only fail embeddedness if the boundary $\Gamma$ bumps into the interior of $\Sigma$ $\left(\varphi\left(\operatorname{int}\left(D^{2}\right)\right)\right.$. Also, $\Gamma$ can only intersect the interior of $\Sigma$ nontransversely; i.e., $\Sigma$ has no transverse self-intersection. A pseudo-area minimizing disk $\Sigma$ is smooth and minimal everywhere except where $\Gamma$ meets the interior of $\Sigma$.

On the other hand, this result is true for more general manifolds, namely homogeneously regular 3-manifolds with sufficiently convex boundary. In Section 6 of $[\mathrm{HS}$, one might find the reasons why we need the conditions of being homogeneously regular and being sufficiently convex to extend these results.

Theorem 3.2. Let $\Gamma$ be a simple closed curve bounding an embedded disk in a homogeneously regular 3-manifold $M$ with sufficiently convex boundary. Then, there exists a disk $\Sigma$ in $M$ with $\partial \Sigma=\Gamma$ such that $\Sigma$ minimizes the area among the embedded disks bounding $\Gamma$. Moreover, $\Sigma$ is embedded in the interior, and it is smooth and minimal everywhere except where the boundary $\Gamma$ meets the interior of $\Sigma$.

Proof. Since Lemma 2.1 is valid for these new ambient manifolds, we can still construct the sequence $\left\{D_{i}\right\}$ as before. By replacing the lemmas we used in the previous theorem with their analogs in the new ambient manifold as in Section 6 of [HS], the same proof would work. 
In other words, this theorem applies to closed 3-manifolds, compact 3-manifolds with sufficiently convex boundary, homogeneously regular noncompact 3-manifolds, and homogeneously regular noncompact 3-manifolds with sufficiently convex boundary.

The structure of the pseudo-area minimizing disks given by the main theorem also tells us how to construct nearby special smoothly embedded disks with the same boundary.

Corollary 3.3. Let $M$ be a closed 3-manifold or a homogeneously regular 3-manifold with sufficiently convex boundary. Let $\Gamma$ be a simple closed curve bounding an embedded disk in $M$. Then, for any given $\epsilon>0$, there exist a smooth, embedded disk $\Sigma_{\epsilon}$ in $M$ with $\partial \Sigma_{\epsilon}=\Gamma$ such that $\left|\Sigma_{\epsilon}\right|<C_{\Gamma}+\epsilon$ and there exists a small region $R$ in $\Sigma_{\epsilon}$ where $|R|<\epsilon$ with $\Sigma_{\epsilon}-R$ is a minimal surface. Here $|$.$| represents the$ area, and $C_{\Gamma}$ is the infimum of the areas of the embedded disks in $M$ with boundary $\Gamma$.

Proof. According to Theorem 3.1, the pseudo-area minimizing disk $\Sigma$ can only fail embeddedness along the coincidence set $\lambda$ where the boundary $\Gamma$ bumps into the interior of $\Sigma$. Hence, for a given $\epsilon$, take a small neighborhood of this segment in $\Sigma$, and push every sheet slightly away from $\lambda$ so that we get a smooth, embedded disk. Since we can choose the neighborhood as small as we want, the corollary follows.

Remark 3.2 (Regularity near the coincidence set and the thin obstacle problem). Another interesting question on the problem is the regularity of $\varphi$ near the coincidence set. This is a well-known problem in the literature and it's known as the Thin obstacle problem or Signorini problem. Clearly, $\varphi$ may not be smooth along the coincidence set, but it may have some regularity when restricted to one side of the coincidence set. In the classical setting of the problem in dimension 2 , let $\Omega$ be a bounded open subset in $\mathbf{R}^{2}$ and $A$ be a line segment in $\Omega$. Let $\psi: A \rightarrow \mathbf{R}$ and $g: \partial \Omega \rightarrow \mathbf{R}$ be given where $g \geq \psi$ on $\partial \Omega \cap A$ and where $\psi, g$ are smooth. Then the question is to find $\varphi: \Omega \rightarrow \mathbf{R}$, where $\left.\varphi\right|_{\partial \Omega}=g$ and $\left.\varphi\right|_{A} \geq \psi$, and $\varphi(\Omega)$ has minimum area. In the classical works $\mathrm{Fr}$, $\mathrm{Ni}$ on the problem, the authors showed the Lipschitz continuity of the solutions in all dimensions, and in dimension 2, Richardson showed that the solutions are $C^{1, \frac{1}{2}}$, which is the optimal regularity Ri]. Recently, Guillen generalized Richardson's result to any dimension Gui].

Hence, when we apply these results to our case, when $M=\mathbf{R}^{3}$, and given that a simple closed curve $\Gamma \subset \mathbf{R}^{3}$ is smooth, then the above results imply that if $\varphi$ is as in the main theorem, then for each local sheet of $\Sigma, \varphi$ is $C^{1, \frac{1}{2}}$ on either side of the coincidence set $\lambda$. Also, when $M$ is a hyperbolic 3-manifold and $\Gamma$ is a geodesic, then Calegari-Gabai's result when applied to our case implies $C^{1}$ regularity of $\varphi$ on either side of the coincidence set (Lemma 1.31 in [CG]).

On the other hand, assuming that $\Gamma$ is smooth enough, it is possible to show that $\varphi$ is $H^{1,2}$ near the coincidence set by arguing as in CG (Section 1.7). In particular, if $\Gamma$ is a smooth simple closed curve in $M$, then it can be shown that $\varphi: D^{2} \rightarrow M$ given in the main theorem is in the Sobolev space $H^{1,2}$; i.e., the derivative $d \varphi$ is defined and $L^{2}$ in the sense of distribution. The idea is basically the same if $\varphi_{i}: D^{2} \rightarrow M$ with $\varphi\left(D^{2}\right)=D_{i}$, where $\left\{D_{i}\right\}$ is the sequence of area minimizing disks constructed in section 3.1 with modified metric. Then each $\varphi_{i}$ induces a conformal structure on $D^{2}$. The $L^{2}$ norm of the derivatives can only 
blow up along a neck pinch. In our case, we work with $D^{2}$, and hence we cannot have a neck pinch by the energy minimizing property of $\varphi_{i}$. So, we get the limit of these conformal structures, and the $L^{2}$ norm of $d \varphi$ can be bounded in terms of the $L^{2}$ norms of $d \varphi_{i}$. In other words, the idea is the same with the argument in [CG] except we work with disks instead of surfaces.

There are also relevant results in the literature concerning the structure of the coincidence set. When $M=\mathbf{R}^{3}$ and $\Gamma$ is analytic, then the coincidence set is a finite union of points and intervals in certain cases [Le, [At] (see Remark 1.32 in CG] ).

\section{Concluding Remarks}

In this paper, we studied the embeddedness question of minimal disks in 3manifolds. Unlike the many results considering the question "for which curves must the area minimizing disks be embedded?" in the literature, we analyzed the structure of the surface which minimizes area among the embedded disks whose boundary is any given simple closed curve. Hence, we showed that among all the embedded disks with fixed boundary, the area minimizer (pseudo-area minimizing disk) exists; however, it may not be an embedded disk. It is a disk in the manifold, and it only fails to be an embedding along the coincidence set where the boundary bumps nontransversely into the interior. Other than this exceptional part, the disk is a smoothly embedded minimal surface. Hence, for any simple closed curve in a 3-manifold $M$, we construct a canonical almost embedded disk in $M$ among the embedded disks bounding the given curve.

Intuitively, one might think of the pseudo-area minimizing disk $\Sigma$ in the following way. Let $D$ be the area minimizing disk $M$ with boundary $\Gamma$. As in Figure $1, D$ may not be embedded, and it might have self-intersection. Then, one can get $\Sigma$ from $D$ by pushing the self-intersection into the boundary. The interesting fact here is that $\Sigma$ is smooth, minimal, and embedded everywhere except where $\Gamma$ meets the interior of $\Sigma$, say $\lambda$. An alternative way to see the picture is that if you push from the coincidence set $\lambda$ in $\Sigma$ into the convex part, you can reduce the area of $\Sigma$ (like a folding curve in [MY2]), but you create a transverse self-intersection in the interior. This cannot happen as $\Sigma$ is minimizing area among the embedded disks. In other words, the coincidence set $\lambda$ behaves like a barrier to embeddedness, even though you can reduce the area by going in that direction.

Also, one might ask whether $\varphi$ is an embedding on the whole $\operatorname{int}\left(D^{2}\right)$ or not. This is not true in general. The reason is that the interior might have nontransverse self-intersection with itself at the coincidence set $\lambda$. For example, if $\Sigma$ has more than one local sheet near the coincidence set $\lambda$, then $\left.\varphi\right|_{\operatorname{int}\left(D^{2}\right)}$ cannot be an embedding. To construct such an example, one can take two "parallel" close embedded area minimizing disks $\Sigma_{1}$ and $\Sigma_{2}$. Then, by adding a tiny bridge $\beta$ between them one can get another area minimizing disk $\Sigma$ with new boundary which is close to $\Sigma_{1} \cup \Sigma_{2} \cup \beta$. Now, if you make a thin and long horn from the part close to $\Sigma_{1}$ which intersects both $\Sigma_{1}$ and $\Sigma_{2}$ transversely as in Figure 1, then the area minimizing disk $\hat{\Sigma}$ which minimizes area for the new boundary curve is an example for such a situation. One needs to push the intersection of the horn with both $\Sigma_{1}$ and $\Sigma_{2}$ to the boundary, and the interiors of them will meet in the boundary. Hence, $\varphi$ cannot be an embedding on $\operatorname{int}\left(D^{2}\right)$ in general. 
On the other hand, there is a relevant result about the same question due to Meeks-Yau [MY3]. They give a necessary condition for a sufficiently smooth simple closed curve in a 3-manifold to bound a strictly stable embedded minimal disk.

Theorem 4.1 ([MY3, Theorem 3]). Let $\Gamma$ be a $C^{2, \alpha}$ simple closed curve in a 3manifold $M$. Then, $\Gamma$ bounds a strictly stable minimal disk $\Sigma$ in $M$ if and only if there exists a sufficiently convex codimension-0 submanifold $N$ in $M$, whose topological type is a 3-ball, and $\Gamma \subset \partial N$.

Hence, by combining this result with ours for $C^{2, \alpha}$ smooth simple closed curves, we conclude that if $\Gamma$ is a $C^{2, \alpha}$ simple closed curve in a 3-manifold $M$, and there is no sufficiently convex domain $N$ as in the above theorem with $\Gamma \subset \partial N$, then the pseudo-area minimizing disk $\Sigma$ given by our main theorem is not embedded up to the boundary. In other words, if $\varphi: D^{2} \rightarrow M$ parameterizes $\Sigma$ with $\varphi\left(D^{2}\right)=$ $\Sigma, \varphi\left(\partial D^{2}\right)=\Gamma$, then $\left.\Gamma \cap \varphi\right|_{\operatorname{int}\left(D^{2}\right)} \neq \emptyset$. However, neither our result nor the above theorem of Meeks-Yau says anything about unstable minimal disks. It is still possible for such a simple closed curve in a 3-manifold $M$ to bound an embedded unstable minimal disk in $M$.

\section{ACKNOWLEDGEMENTS}

The author would like to thank Danny Calegari, David Gabai and Joel Hass for very helpful comments. He also thanks the referee for very valuable suggestions.

\section{REFERENCES}

[Al] H.W. Alt, Verzweigungspunkte von H-Flachen. II, Math. Ann. 201 (1973), 33-55. MR0331195 (48:9529)

[AS] F.J. Almgren and L. Simon, Existence of embedded solutions of Plateau's problem, Ann. Scuola Norm. Sup. Pisa Cl. Sci. (4) 6 (1979) no. 3, 447-495. MR.553794 (81d:49025)

[At] I. Athanasopoulos, Coincidence set of minimal surfaces for the thin obstacle, Manuscripta Math. 42 (1983) 199-209. MR.701203 (85a:49048)

[CG] D. Calegari and D. Gabai, Shrinkwrapping and the taming of hyperbolic 3-manifolds, J. Amer. Math. Soc. 19 (2006), no. 2, 385-446. MR2188131 (2006g:57030)

[CM] T. Colding and W.P. Minicozzi, Minimal surfaces, Courant Lecture Notes in Mathematics, 4. New York, 1999. MR1683966 (2002b:49072)

[Do] J. Douglas, Solution of the problem of Plateau, Trans. Amer. Math. Soc. 33 (1931) 263321. MR 1501590

[EWW] T. Ekholm, B. White, and D. Wienholtz, Embeddedness of minimal surfaces with to-

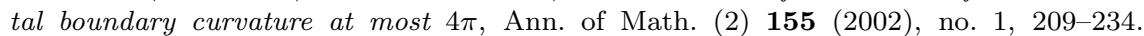
MR1888799 (2003f:53010)

[Fe] H. Federer, Geometric measure theory, Springer-Verlag, New York, 1969. MR0257325 (41:1976)

[Fr] J. Frehse, Two dimensional variational problems with thin obstacles, Math. Z. 143 (1975), no. 3, 279-288. MR0380550 (52:1450)

[Ga] D. Gabai, On the geometric and topological rigidity of hyperbolic 3-manifolds, J. Amer. Math. Soc. 10 (1997) 37-74. MR1354958 (97h:57028)

[GS] R. Gulliver and J. Spruck, On embedded minimal surfaces, Ann. of Math. (2) 103 (1976) 331-347. MR0405217 (53:9011)

[Gu] R.D. Gulliver, Regularity of minimizing surfaces of prescribed mean curvature, Ann. of Math. (2) 97 (1973) 275-305. MR0317188(47:5736)

[Gui] N. Guillen, Optimal regularity for the Signorini problem, Calc. Var. Partial Differential Equations 36 (2009), 533-546. MR2558329 (2010j:35619)

[HLT] J. Hass, J.C. Lagarias and W.P. Thurston, Area inequalities for embedded disks spanning unknotted curves, J. Differential Geom. 68 (2004) no. 1, 1-29. MR2152907|(2006e:53015) 
[HS] J. Hass and P. Scott, The existence of least area surfaces in 3-manifolds, Trans. Amer. Math. Soc. 310 (1988) no. 1, 87-114. MR965747 (90c:53022)

[Le] H. Lewy, On the coincidence set in variational inequalities, J. Differential Geometry 6 (1972) 497-501. MR0320343 (47:8882)

[Mo] C.B. Morrey, The problem of Plateau on a Riemannian manifold, Ann. of Math. (2) 49 (1948) 807-851. MR0027137 (10:259f)

[MY1] W. Meeks and S.T. Yau, Topology of three-dimensional manifolds and the embedding problems in minimal surface theory, Ann. of Math. (2) 112 (1980) 441-484. MR.595203 (83d:53045)

[MY2] W. Meeks and S.T. Yau, The classical Plateau problem and the topology of three manifolds, Topology 21 (1982) 409-442. MR670745 (84g:53016)

[MY3] W. Meeks and S.T. Yau, The existence of embedded minimal surfaces and the problem of uniqueness, Math. Z. 179 (1982) no. 2, 151-168. MR645492 (83j:53060)

[Ni] J.C.C. Nitsche, Variational problems with inequalities as boundary conditions or how to fashion a cheap hat for Giacometti's brother, Arch. Rational Mech. Anal. 35 (1969) 83113. MR.0248585 (40:1837)

[Os] R. Osserman, A proof of the regularity everywhere of the classical solution to Plateau's problem, Ann. of Math. (2) 91 (1970) 550-569. MR0266070 (42:979)

[Ra] T. Rado, On Plateau's problem, Ann. of Math. (2) 31 (1930) no. 3, 457-469. MR.1502955

[Ri] D. Richardson, Variational problems with thin obstacles, Ph.D. Thesis, The University of British Columbia (1978). MR2628343

[TT] F. Tomi and A.J. Tromba, Extreme curves bound embedded minimal surfaces of the type of the disc, Math. Z. 158 (1978) 137-145. MR486522 (80k:53011)

Department of Mathematics, Koc University, Sariyer, Istanbul 34450 Turkey

E-mail address: bcoskunuzer@ku.edu.tr 